\title{
Performance Analysis of Double Channel SW-ARQ System
}

\author{
LI Suoping ${ }^{\text {a }}$, BAI Zhongyu , LIU Mei \\ School of Science, Lanzhou University of Technology, Lanzhou 730050, China \\ alsuop@163.com
}

Keywords: SW-ARQ system, Markov chain, Double channel, Packet average delay, Throughput Abstract. An double SW-ARQ system model has been established for wireless communication in this paper. We analyzed the packet average delay for the system by an alternative method of retransmission, and recognize the packet's number in the buffer at receiver as the initial state of the system, the final state of the buffer reaches the channel's number by a limited times of retransmissions. For this complete transmission process, the packet average delay formula is obtained based on Markov state transition probability matrix, and make a comparative study how to control the optimal packet length to guarantee the largest throughput.

\section{Introduction}

ARQ(automatic repeat request)technology with reliable data transmission has become widely used in errors control technology[1]. [2] analyzed ARQ system performance when the size of buffer at the receiver is not the same. [3] proposed Multichannel SW-ARQ (MSW-ARQ) model and analyzed packet transmission delay. Because the buffer of receiver is not considered in these papers, so which undoubtedly cause an increasing of average packet delay. [4] investigated the throughput and energy efficiency of multiple sources multiple relays cooperative ARQ (MSMR-CARQ) system in wireless sensor networks. In [5], by analyzing the transmission mechanism of SR-ARQ(Selective-Repeat ARQ), which get the expression formulations of the packet mean waiting delay by solving Markov chain. [6] investigated the performance of multicast cooperative ARQ (MCARQ) in wireless networks, then get the steady-state distribution of the MCARQ by solving the Markov chain. [7] investigated the power allocation scheme based on cooperative system with MIMO-OFDM.

We make the following rules; the sender has a steady stream of packets needed to be sent. $M$ denotes the number of final state of buffer. $p$ denotes the transmission error rate and loss rate of packets. $P\left[\xi_{i}=j\right]$ denotes the probability of state which from 0 to $i$ and the packet has experienced $j$ retransmission using time $j m+\left(K_{f} / R\right)$. $\eta$ denotes the system throughput. $K_{f}$ denotes the number of bits of each packet contained. $R$ denotes the transmission speed. Assuming that the length of each packet is same, and the delay of sender, detection delay of receiver and feedback delay are neglected.

\section{Single Channel SW-ARQ System and its Performance Analysis}

\subsection{Single channel SW-ARQ system}

The principle of signal channel transmission of SW-ARQ system is shown in Fig.1. Where S denotes sender, $\mathrm{R}$ denotes receiver. The sender sendes packets to recriver by channel. And then, the receiver sendes feedback packets to sender by the same channel.

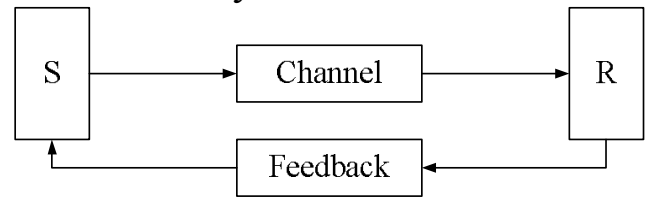

Fig. 1 Principle of signal channel transmission of SW-ARQ. 
The Transmission process of single channel SW-ARQ system can be described as shown in Figure 2. Starting with $\operatorname{tm}(t=0,1,2 \ldots n)$, the sender send a packet to the receiver. The receiver receives packet at the time of $t m+(m-1) / 2$, and after error detection, the receiver sendes ACK or NACK, these actions are finished at the end of the slot of $t m+(m-1) / 2$.

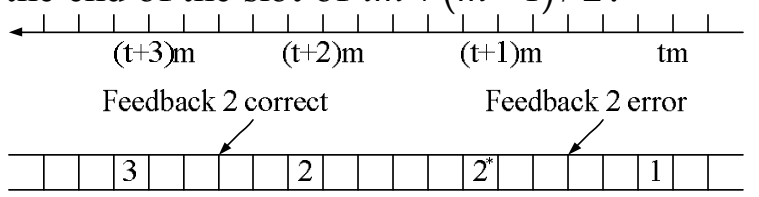

Fig. 2 transmission process of SW-ARQ $(m=5 . *$ denotes transmission error and packet loss $)$

\subsection{Modelling of single channel SW-ARQ system}

Assuming that channel number is $M$, and $M=1$ means there is only one channel for communication. Due to a packet's success is closely related with the former, so we can use markov chain's method to analyze it, which has the state space $\{0,1\}$, we can get the single channel state transition probability matrix. We denote the state transition probability matrix of markov chain by $P_{M=1}, p_{00}=p$ denotes the probability of no packet being transmtted successfuly, given $p_{11}=0 \cdot p_{0 i}=C_{n}^{i}(1-p)^{i} p^{n-i}$ ( $i=0,1, n=1$ ) denotes the probability of state from 0 to $i \cdot p_{10}=1$ denotes the probability of buffer being filled, and all packets will be submitted to the upper, and then buffer state becomes 0 . that is because the packet is submitted immediately to the upper when the buffer is filled with packet, and then the buffer is released. The delay of submittion and feedback packet are neglected.

By the method of mathematical expectation, we can get the packet average delay of single SW-ARQ system as foolows

$$
E\left[T_{1}(k)\right]=\sum_{k=0}^{n}\left(\left[k m+\left(\frac{K_{f}}{R}\right)\right] \cdot P\left[\xi_{1}=k\right]\right) .
$$

Based on the system throughput formula in [2], we can get

$$
\eta_{1}=\frac{K_{f} / R}{E\left[T_{1}(k)\right]}=\frac{\left(K_{f} / R\right)}{\sum_{k=0}^{n}\left(\left[k m+\left(\frac{K_{f}}{R}\right)\right] \cdot P\left[\xi_{1}=k\right]\right)}
$$

Where $P\left[\xi_{1}=n\right]=P\left[\xi_{1}=n-1\right] p_{00}$.

\section{Double Channel SW-ARQ System Model and its Performance Analysis}

3.1 Transmission process of double channel SW-ARQ system

The transmission principle of double channel SW-ARQ system can be shown as in Fig.3.

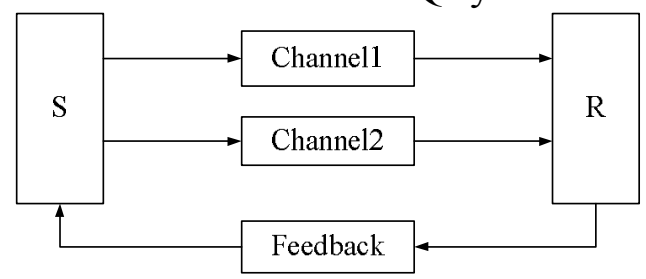

Fig. 3 Transmission principle of double channel SW-ARQ

The transmission process is shown in figure 4. Starting with the time of $\operatorname{tm}(t=0,1,2 \ldots n)$, the sender sendes two packets to the receiver, the sender receives ACKs or NACKs at the end of $t m+(m-1)$, The receiver received packets in slot $t m+(m-1) / 2$. The receiver sends ACKs or NACKs through the error detection. The actions are finished at the end of the slot $t m+(m-1) / 2$. 


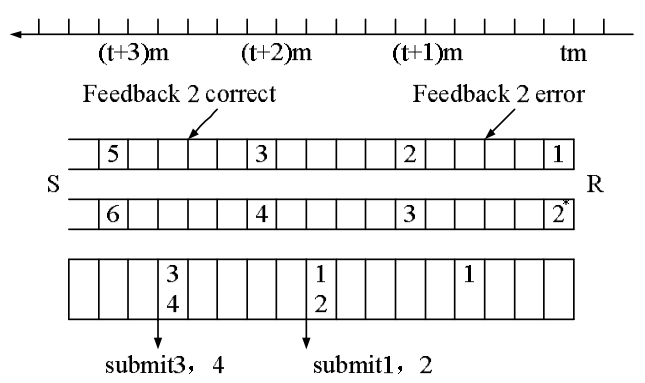

Fig. 4 Transmission process,$(m=5)$

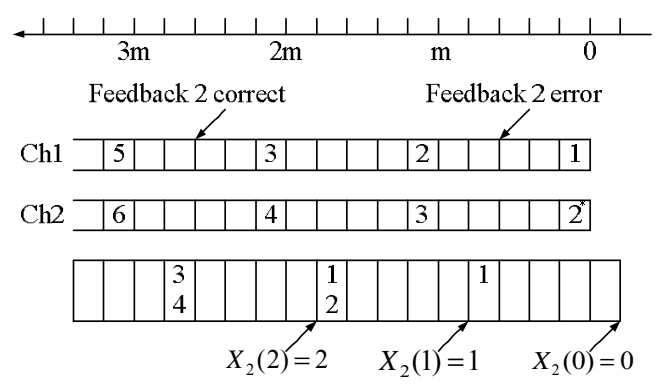

Fig. 5 The state transition process $(m=5 \mathrm{~s})$

3.2 Transmission analysis of double channel SW-ARQ system

Let the double channel SW-ARQ system has the state transition probability matrix on state space $\{0,1,2\}$. Denote the matrix of markov chain by $P_{M=2}$. Let $p_{00}=p^{2}$ denote the probability of the two unsuccessfully transmitted packets. given $p_{22}=0 . p_{0 i}=C_{n}^{i}(1-p)^{i} p^{n-i}(i=0,1,2, n=2)$ denotes the probability of state from 0 to $i, p_{i i}=p^{n-i}(i<n)$ denotes the probability which the system state number in the adjacent two slots has not been changed. $p_{i j}=0(j<i<2)$ denotes the probability which the packets could not be submitted to the upper if the buffer is not filled, when the buffer is filled with two packets, and then it is submitted to the upper immediately, then the buffer space is released. $p_{20}=1$ represents that the buffer is just filled, and all packets will be submitted to the upper, and then buffer state becomes 0 . The delays of submittion and feedback are neglected.

Through the method of mathematical expectation, we can conclude the packet average delay of double channel SW-ARQ system as follows

$$
E\left[T_{2}(k)\right]=\sum_{k=0}^{n}\left(\left[k m+\left(\frac{K_{f}}{R}\right)\right] \cdot P\left[\xi_{2}=k\right]\right) .
$$

Based on the system throughput formula in [2], we can get

$$
\eta_{2}=\frac{\left(K_{f} / R\right) / 2}{\sum_{k=0}^{n}\left(\left[k m+\left(\frac{K_{f}}{R}\right)\right] \cdot P\left[\xi_{2}=k\right]\right)} .
$$

Where

$$
\begin{aligned}
& P\left[\xi_{2}=n\right]=P\left[x_{2}(n)=2, x_{2}(n-1) \neq 2, \ldots, x_{2}(1) \neq 2 \mid x_{2}(0)=0\right] \\
& =\sum_{j=0}^{2} P\left[x_{2}(n)=2, x_{2}(n-1) \neq 2, \ldots, x_{2}(2) \neq 2 \mid x_{2}(1)=j\right] p_{0 j} \\
& =\sum_{j=0}^{2} P\left[\xi_{j}=n-1\right] p_{0 j}
\end{aligned}
$$

\section{Simulation and results}

Given $K_{f} / R=0.2$ as the transmission delay, limited $n=100$ as the retransmission times, through computer simulation, we can get the simulation graph. In figure 6 and7, we set the channel error rate and packets loss rate increase from 0.1 to 0.5 , if we do not consider other factors, we can see from the figure 6 by simulation that the packets delay of single channel will increase with the increasing of error and packets loss rate, and the throughput of SW-ARQ system will decrease with the increasing of rate of error and loss. In figure 7 , we also set $p$ increase from 0.1 to 0.5 , under the condition of the same error rate, To further analysis, as shown in figure 7, From the figure, we find that the packet average delay of single channel SW-ARQ system is the largest when $p$ less than 0.3 , but, when when $p$ more than 0.3 , the throughput of double channel SW-ARQ system less than single channel. 

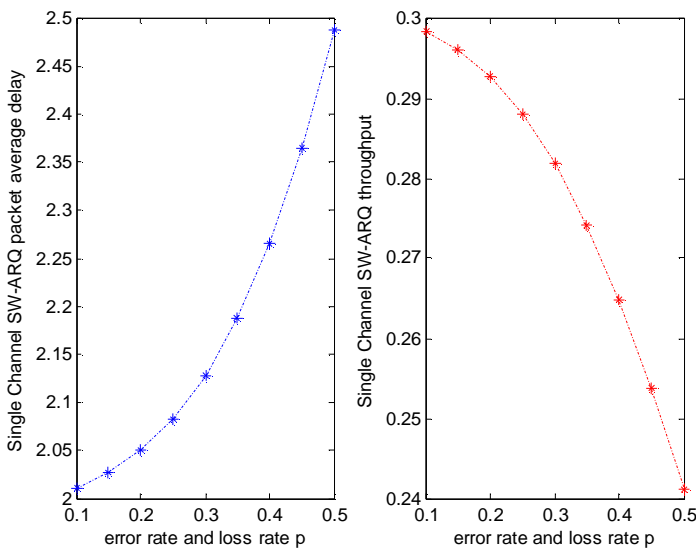

Fig. 6 Performance of single system $(\mathrm{m}=1)$
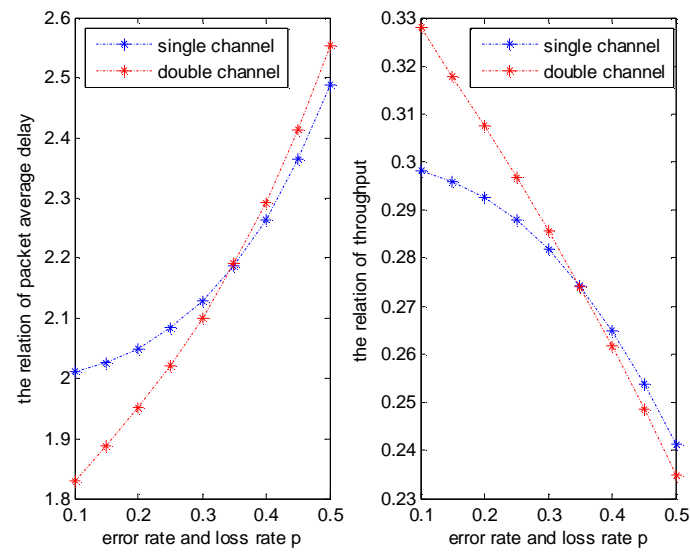

Fig. 7 Reltion of single and double system $(\mathrm{m}=1)$

\section{Conclusion}

An double SW-ARQ system model has been established in this paper. We get the average packet delay of single channel SW-ARQ and double channel SW-ARQ system by solving Markov model. Further work is that we can dynamically distribute different packet under the different conditions of channel according to the packet length. By solving the Markov model, we get the average delay of the system and make a comparative study of how to control the optimal packet length to guaranteen the largest throughput.

\section{References}

[1] Pingyi Fan, Chongxi Feng. Fundamentals of modern communication theory(Zhong ce)networking technology, first ed., Tsinghua University Press, 2007.

[2] H. Jianhua, K. R. Rubramanian, Novel methods for the performance analysis of adaptive hybird selective repeat ARQ, J. Computer Communication, 23 (2000) 1548 -1557.

[3] Jun Li, Yiqiang Q. Zhao. Packet delay analysis for multichannel communication systems with MSW-ARQ, J. Performance Evaluation, 66 (2009) 380-394.

[4] Yongqiang Zhou, Suoping Li, Shanglin Hou. Throughput and Energy Efficiency of Multiple Sources Multiple Relays Cooperative ARQ System for WSNs, J. Journal of Computational Information System, 8 (2012) 1-8.

[5] Suoping Li, Yongqiang Zhou. Performance Analysis of SR-ARQ Based on Geom/G/1/œQueue over Wireless Link, J. Applied Mathematics \& Information Sciences, 7 (2013) 1969-1976.

[6] Suo Ping Li, Yong Qiang Zhou, Yong Zhou. Delay and energy efficiency analysis of multicast cooperative ARQ over wireless networks, J. Acta Information, 51 (2014) 51-60.

[7] Suoping Li, Xuewei Zou, Lifu Yan, Power Allocation Scheme for Channel Capacity Maximization in Multi-relay Amplify-and-Forward Cooperative System, J. Journal of Information \& Computational Science, 12 (2015) 1365-1372. 